\title{
Novel Oil-in-Water Emulsions Stabilised by Ionic Surfactant and Similarly Charged Nanoparticles at Very Low Concentrations
}

\author{
M.D. Xu, ${ }^{[a]}\left[{ }^{[b]} J . Z . ~ J i a n g,{ }^{[a]}\right.$ X.M. Pei, ${ }^{[a]}$ B.L. Song, ${ }^{[a]}$ Z.G. Cui ${ }^{*[a]}$ and B.P. Binks ${ }^{\star[c]}$
}

\begin{abstract}
Novel oil-in-water $(\mathrm{O} / \mathrm{W})$ emulsions are prepared which are stabilised by a cationic surfactant in combination with similarly charged alumina nanoparticles at concentrations as low as $10^{-5} \mathrm{M}$ and $10^{-4} \mathrm{wt} . \%$, respectively. The surfactant molecules adsorb at the oil-water interface to reduce the interfacial tension and endow droplets with charge ensuring electrical repulsion between them, whereas the charged particles are dispersed in the aqueous films between droplets retaining thick lamellae, reducing water drainage and hindering flocculation and coalescence of droplets. This stabilization mechanism is universal as it occurs with different oils (alkanes, aromatic hydrocarbons and triglycerides) and in mixtures of anionic surfactant and negatively charged nanoparticles. Further, such emulsions can be switched between stable and unstable by addition of an equimolar amount of oppositely charged surfactant which forms ion pairs with the original surfactant destroying the repulsion between droplets.
\end{abstract}

It is well known that conventional emulsions are stabilised by surfactants or polymers. ${ }^{[1]}$ Their molecules adsorb at the oilwater interface to reduce the interfacial tension (IFT) and prevent flocculation and coalescence of droplets via either electrostatic or steric repulsion or both. ${ }^{[1,2]}$ These emulsions, however, are thermodynamically unstable and it is common that a minimum surfactant concentration close to the critical micelle concentration $(\mathrm{cmc})$ is required to ensure kinetic stabilization. ${ }^{[1,2]}$

In contrast, the so-called Pickering emulsions stabilised by colloid particles are usually much more stable, in which particles adsorb or aggregate at the oil-water interface forming a solid shell preventing coalescence. ${ }^{[3-5]}$ Surface-active particles may be either amphiphilic (asymmetric) called Janus particles, ${ }^{[6-13]}$ or possess homogeneous surfaces such as modified inorganic particles [14-17] and various organic particles with or without grafted functional groups. ${ }^{[18]}$ Some of the latter are switchable between being surface-active and surface-inactive using stimuli such as $\mathrm{pH}$, temperature and $\mathrm{CO}_{2} / \mathrm{N}_{2}$ addition. ${ }^{[18]}$

A question which arises is what will happen when surfactant molecules meet particles in an emulsion system? Arab et al. have just given a review on this topic, ${ }^{[19]}$ and up to now systems

[a] Mr. MD Xu, Dr. JZ Jiang, Dr. XM Pei, Dr. BL Song, Prof. ZG Cui The Key Laboratory of Synthetic and Biological Colloids, Ministry of Education, School of Chemical and Material Engineering, Jiangnan University, 1800 Lihu Road,

Wuxi, Jiangsu 214122, P.R. China

cuizhenggang@hotmail.com

[b] Mr. MD Xu

School of Biological and Chemical Engineering

Anhui Polytechnic University,

Wuhu 241000, P.R. China

[c] Prof. BP Binks

School of Mathematics and Physical Sciences

University of Hull,

Hull. HU6 7RX. U.K.

b.p.binks@hull.ac.uk

Supporting information for this article is given via a link at the end of the document involving ionic surfactants and oppositely charged inorganic particles have been widely studied, ${ }^{[19-28]}$ in which hydrophilic particles like silica, ${ }^{[19,21,23,24]}$ laponite, ${ }^{[20]}$ or calcium carbonate [15,22] can be made surface-active by adsorbing oppositely charged ionic surfactant so as to stabilize a Pickering emulsion. Depending on the type and adsorbed amount of surfactant, the Pickering emulsions can undergo a single or double phase inversion ${ }^{[15,22,23]}$ and even be made switchable ${ }^{[25]}$ or stimuliresponsive. ${ }^{[26-29]}$

On the other hand, emulsion systems involving similarly charged ionic surfactant and particles are much less studied. [19,30-32] Herein we report for the first time novel O/W emulsions which can be stabilised by a cationic surfactant (cetyltrimethyl ammonium bromide, CTAB) in combination with positively charged alumina nanoparticles at extremely low concentration (ca. $0.001 \mathrm{cmc}$ and $0.0001 \mathrm{wt} \%$, respectively). Surfactant alone stabilises emulsions (at much higher concentrations) whereas particles do not. The droplet size in mixtures is comparable to that in emulsions stabilised by CTAB alone at relatively high concentration, and the emulsions can be switched between stable and unstable on addition of an anionic surfactant (sodium dodecyl sulfate, SDS). The findings are applicable to various oils like alkanes, aromatic hydrocarbons and triglycerides and appear to be universal occurring for cationic surfactants with positively charged particles and anionic surfactants with negatively charged particles.

High purity alumina nanoparticles $\left(\mathrm{Al}_{2} \mathrm{O}_{3}\right.$, purity $\left.>99.8 \%\right)$ have a primary diameter of $13 \mathrm{~nm}$ and a measured BET surface area of $89.8 \pm 1.2 \mathrm{~m}^{2} / \mathrm{g}$ (see SEM and TEM images in Fig. S1). They form aggregates of average diameter $\left(\mathrm{d}_{\mathrm{av}}\right) 192 \mathrm{~nm}$ (Fig S2) and possess a zeta potential of $+55 \mathrm{mV}$ when dispersed in pure water $\left(\mathrm{pH} \approx 6\right.$, Fig. S3). The value of $d_{a v}$ is not changed $(198 \pm 4 \mathrm{~nm})$ when dispersed in aqueous CTAB solutions (0.001-1.0 mM). Using $n$-decane (> 98\%) as oil phase $(1: 1$ by vol.), the particles alone do not stabilize an emulsion, nor does CTAB alone at concentrations lower than $0.6 \mathrm{mM}(\mathrm{cmc}=0.9$ $\mathrm{mM}{ }^{[24]}$ ) as shown in Fig. S4. However, when alumina nanoparticles (0.5 wt.\%) were dispersed in aqueous CTAB solutions, stable $\mathrm{O} / \mathrm{W}$ emulsions can be prepared at $\mathrm{CTAB}$ concentrations well below $0.6 \mathrm{mM}$. These emulsions remained stable to coalescence upon standing at room temperature for at least 1 month, as shown in Figure 1(A). Selected micrographs (taken $24 \mathrm{~h}$ after preparation) shown in Figure 1(B) indicate that except at $0.01 \mathrm{mM} \mathrm{CTAB}$ where relatively large droplet sizes were observed $(B-1)$, the droplet sizes at all other $C T A B$ concentrations are relatively small $(20-60 \mu \mathrm{m})$ and decrease slightly with increasing CTAB concentration (B-2, B-3) and are close to those in emulsions stabilised by CTAB alone at similar concentrations (0.6-2.0 mM, Figure $1(\mathrm{C})$ ). Remarkably, in the case of a fixed low CTAB concentration of $0.01 \mathrm{mM}$, stable $n$ decane-in-water emulsions can be formed at alumina concentrations as low as $3 \times 10^{-4}$ wt.\%, with droplet sizes between 40 and $100 \mu \mathrm{m}$ as shown in Figure 2. The volume fraction of oil in the creamed emulsions reaches $73 \pm 1 \%$.

This is the peer reviewed version of the following article: M. Xu, J. Jiang, X. Pei, B. Song, Z. Cui, B. P. Binks, Angew. Chem. Int. Ed. 2018, 57, 7738., which has been published in final form at https://doi.org/10.1002/anie.201802266. This article may be used for non-commercial purposes in accordance with Wiley Terms and Conditions for Use of Self-Archived Versions. 
The synergistic effect of alumina nanoparticles and CTAB, both positively charged in water, in stabilizing $n$ - decane-in-water emulsions is obvious. Figure 3 shows the correlation between

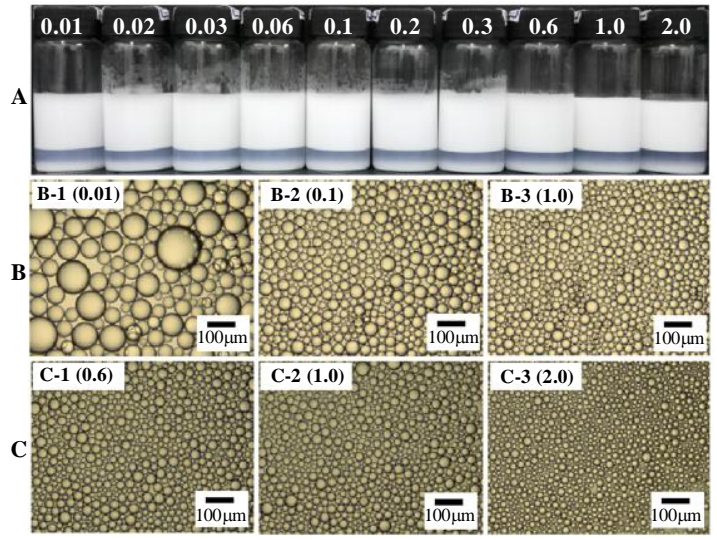

Figure 1. (A) Digital photographs and (B) selected micrographs of $n$-decanein-water emulsions $(7 \mathrm{~mL} / 7 \mathrm{~mL})$ stabilised by mixtures of $0.5 \mathrm{wt} . \%$ alumina and CTAB of different concentration, as well as (C) selected micrographs of $n$ decane-in-water emulsions $(7 \mathrm{~mL} / 7 \mathrm{~mL})$ stabilised by CTAB alone at different concentration, taken $24 \mathrm{~h}$ ( $\mathrm{B}$ and $\mathrm{C}$ ) and 1 month $(\mathrm{A})$ after preparation. $[\mathrm{CTAB}] / \mathrm{mM}$ is given on each vessel and in each image.

the minimum concentration of alumina nanoparticles $\left(\mathrm{C}_{\min , \mathrm{Al} 2 \mathrm{O} 3}\right)$ and the minimum concentration of $\mathrm{CTAB}\left(\mathrm{C}_{\mathrm{min}, \mathrm{CTAB}}\right)$ required for preparing a stable $n$-decane-in-water emulsion (a), and the average droplet size of the corresponding stable emulsions (b) based on micrographs shown in Fig. S5. It can be seen that an overall minimum occurs at 0.0001 wt.\% alumina and $0.01 \mathrm{mM}$ $\mathrm{CTAB}$. The value of $\mathrm{C}_{\text {min, } \mathrm{Al} 2 \mathrm{O} 3}$ decreases with increasing $\mathrm{CTAB}$ concentration on the left of the minimum, but increases with increasing $C T A B$ concentration on the right of the minimum. However, the average droplet size decreases monotonically with increasing $\mathrm{CTAB}$ concentration, approaching that in emulsions stabilised by CTAB alone at higher concentration (from Figure 1 , C-1 - C-3) as shown in Figure 3(b).

We reported recently that stable $n$-decane-in-water Pickering emulsions with much larger droplet size $(50-200 \mu \mathrm{m})$ can be prepared by using cationic alumina nanoparticles plus an anionic surfactant SDS (0.02-0.2 mM) as emulsifier, ${ }^{[26]}$ as shown in Fig. S6, where the alumina nanoparticles are hydrophobized in situ by adsorption of SDS molecules and are then capable of adsorbing at the oil-water interface. It is natural to ask what is the microstructure of emulsions stabilised by alumina nanoparticles in combination with a trace amount of CTAB? Are they classical Pickering emulsions or conventional emulsions stabilised solely by surfactant? If the latter, how do the particles enhance emulsion stability?

By measuring the concentration of alumina nanoparticles in the resolved aqueous phase of emulsions after creaming (Table $\mathrm{S} 1)$, it is found that in systems with CTAB $(0.01-1.0 \mathrm{mM})$ the particle concentration is only slightly lower than the initial concentration, suggesting that no particles adsorb to the oilwater interface of emulsion drops (we acknowledge that a low \% of particles will be in the aqueous phase of the emulsion

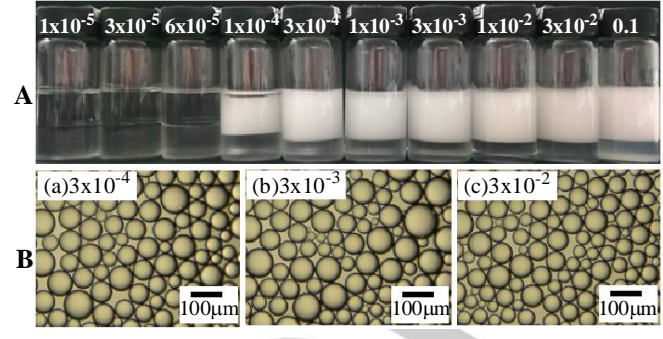

Figure 2. (A) Digital photographs of $n$-decane-in-water emulsions $(7 \mathrm{~mL} / 7 \mathrm{~mL})$ stabilised by $0.01 \mathrm{mM} \mathrm{CTAB}$ in combination with alumina nanoparticles at different concentration (given on each vessel in wt.\%), (B) selected micrographs of emulsions, taken one week (A) and $24 \mathrm{~h}(\mathrm{~B})$ after preparation.
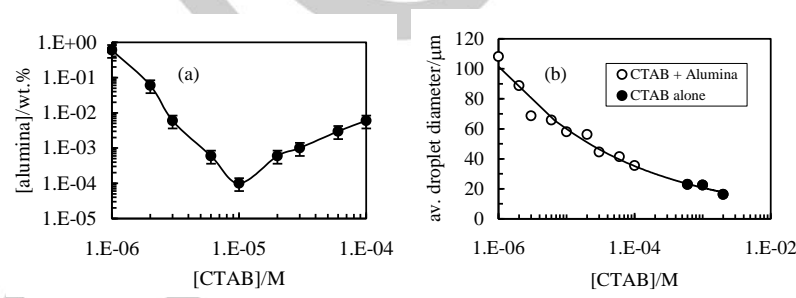

Figure 3. (a) Minimum alumina concentration required to stabilize $n$-decane in-water emulsions as a function of CTAB concentration at room temperature, (b) average droplet diameter of corresponding emulsions and those stabilised by $C T A B$ alone as a function of CTAB concentration measured from micrographs given in Fig. S5 and Fig. 1(C-1 to C-3), respectively. The solid line in (b) is a guide for the eye.

however). In contrast, in systems with SDS (Pickering emulsion) the particle concentration decreases significantly from 0.500 wt.\% (initial) to $0.016 \mathrm{wt} . \%$ in the presence of $1.0 \mathrm{mM}$ SDS (< $\mathrm{cmc}$ ). This result, together with the difference in droplet sizes in the two systems, implies that the droplets in systems with CTAB are stabilised by CTAB molecules with alumina nanoparticles remaining dispersed in the intervening aqueous phase.

This is further proved by the SEM images and optical micrographs of the emulsion droplets (with $n$-decane replaced by the more volatile $n$-hexane) shown in Figure 4 . It is seen clearly that in the case of $0.5 \mathrm{wt} \%$ alumina plus $0.1 \mathrm{mM} \mathrm{CTAB}$ the dried droplets appear as spherical holes (oil evaporated) with alumina particles distributed in the continuous phase (Figure $4 \mathrm{~A}-1$ and $\mathrm{B}$ ). The same scenario is also observed at a lower concentration of CTAB, $0.03 \mathrm{mM}$, and particles, $0.06 \mathrm{wt} . \%$ (Figure $4 \mathrm{~A}-2)$. The few particles appearing within the circle (A-1) arise from the aqueous film which covered the droplet or from close by due to air flow during drying. In contrast, in the case of alumina nanoparticles plus SDS, the half dried droplets (Figure 4 C-1) possess a wrinkled skin and adopt non-spherical shapes on drying, leaving solid film stamps when fully dried (Figure 4C-2). In summary, we believe that $n$-decane-in-water emulsions containing alumina and CTAB are not Pickering emulsions but rather emulsions stabilised by cationic surfactant at the oil-water interface with nanoparticles dispersed in the aqueous films between droplets. 


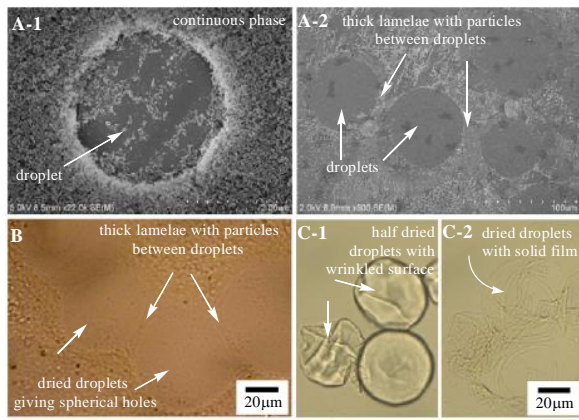

Figure 4. (A) SEM images and (B) optical micrographs of dried $n$-hexane-inwater emulsion droplets stabilised by 0.5 wt.\% alumina nanoparticles plus 0.1 $\mathrm{mM} \mathrm{CTAB}(\mathrm{A}-1, \mathrm{~B})$ or by 0.06 wt.\% alumina nanoparticles plus $0.03 \mathrm{mM}$ CTAB (A-2) and (C) optical micrographs of $n$-hexane-in-water emulsion droplets half dried (C-1) and fully dried (C-2) stabilised by 0.5 wt.\% alumina nanoparticles plus $0.1 \mathrm{mM}$ SDS, taken $24 \mathrm{~h}$ after preparation. A-2 and B: lamelae should be lamellae

This stabilization mechanism also operates when different oils like alkanes, aromatic hydrocarbons or triglycerides were used as the oil phase. For toluene and tricaprylin, stable O/W emulsions can be formed using 0.5 wt. $\%$ alumina nanoparticles plus CTAB at as low as $0.003 \mathrm{mM}$ and $0.0006 \mathrm{mM}$ respectively, as shown in Fig. S7 and Fig. S8. Although droplet diameters are relatively large $(100-200 \mu \mathrm{m})$ at such a low CTAB concentration, they can be reduced to as low as $30 \mu \mathrm{m}$ by increasing the CTAB concentration to $0.06 \mathrm{mM}$. These emulsions are also stable to coalescence at room temperature for over 6 months. Importantly this phenomenon is also applicable to negatively charged silica nanoparticles (0.5 wt.\%) and anionic surfactant SDS as shown in Fig. S9, where the SDS concentration can be as low as $0.0001 \mathrm{mM}(\mathrm{cmc}=8 \mathrm{mM})$ and emulsions are again stable to coalescence for up to at least 6 months.

The non-adsorption of CTAB on alumina nanoparticles and of alumina nanoparticles at the oil-water interface is also supported by the zeta potential and surface tension (ST)/IFT data shown in Fig. S10 and Fig. S11, respectively. The zeta potential decreases slightly from $+55 \mathrm{mV}$ in pure water to +41 $\mathrm{mV}$ in the presence of $1 \mathrm{mM}$ CTAB at $25^{\circ} \mathrm{C}$, probably due to an increase in the ionic strength of the aqueous phase, and no flocculation of particles was observed in the dispersions. Both the ST and the decane-water IFT are only slightly lower than that of $C T A B$ solutions without particles, suggesting little reduction of the CTAB monomer concentration in the dispersions due to adsorption onto particle surfaces. Particles therefore remain hydrophilic and surface-inactive $(\mathrm{ST}=71.7 \pm 0.1 \mathrm{mN} / \mathrm{m}$ for particle dispersions alone in pure water at $25^{\circ} \mathrm{C}, 0.0001-0.5$ wt.\%). As in conventional emulsions, the average droplet diameter $\left(D_{a v}\right)$ correlates with the oil-water IFT $(\gamma)$ as shown in Fig. S11 such that $D_{a v}(\mu \mathrm{m}) \propto(5.86 \pm 1.00) \gamma^{0.6}$. ${ }^{[1]}$ Surfactant behaves as the emulsifier whereas particles behave as an additional stabilizer. By contrast, the zeta potential of the particles in SDS solutions decreases significantly and changes sign with increasing SDS concentration, in line with adsorption of SDS at the particle-water interface and subsequent stabilization of Pickering emulsions. ${ }^{[26]}$

The excellent stability of emulsions at very low $(<0.6 \mathrm{mM})$ $\mathrm{CTAB}$ concentration can then be attributed to the presence of non-adsorbed particles. By measuring the lifetime of a single $n$ decane droplet $(2 \mu \mathrm{L})$ at a planar $n$-decane-water interface containing an adsorbed CTAB monolayer (a good measure of droplet stability), it is found that in the absence of particles the droplet lifetime is very short $(<10 \mathrm{~s})$ at CTAB concentrations below $0.06 \mathrm{mM}$ and increases to several $\mathrm{min}$ by $0.3 \mathrm{mM}$. However, in the presence of $0.5 \mathrm{wt} . \%$ particles in the aqueous phase, the lifetime increases dramatically at CTAB concentrations beyond $0.003 \mathrm{mM}$, as shown in Figure 5(a). If we take the CTAB concentration at the break point of the curves as $\mathrm{C}_{\text {min,CTAB, }}$ it decreases from $0.06 \mathrm{mM}$ in the absence of alumina particles to $0.001 \mathrm{mM}$ in the presence of $0.5 \mathrm{wt} . \%$ alumina particles. In
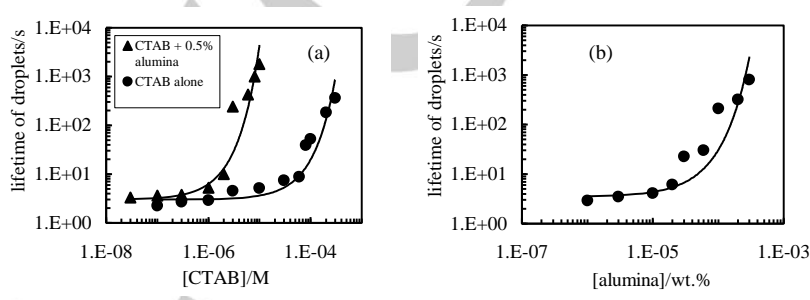

Figure 5. (a) Lifetime of $n$-decane droplets at the pre-equilibrated $n$-decaneaqueous CTAB planar interface with and without 0.5 wt.\% alumina nanoparticles dispersed in the aqueous solution as a function of surfactant concentration at $25^{\circ} \mathrm{C}$, (b) Lifetime of $n$-decane droplets as a function of alumina particle concentration in the presence of $0.01 \mathrm{mM} \mathrm{CTAB}$ at $25^{\circ} \mathrm{C}$.

reality, $\mathrm{C}_{\text {min,CTAB }}$ falls from $0.6 \mathrm{mM}$ for CTAB alone (Fig. S4B) to $0.001 \mathrm{mM}$ should this be $0.01 \mathrm{mM}$ ? in the presence of $0.5 \mathrm{wt} . \%$ particles (Figure $1 \mathrm{~A}$ and Figure 3 ), in good agreement with droplet lifetime measurements. At a fixed CTAB concentration of $0.01 \mathrm{mM}$, the droplet lifetime increases with increasing particle concentration, giving a critical particle concentration of $2 \times 10^{-5}$ wt.\%, Figure 5(b), beyond which the droplet lifetime increases dramatically and emulsions are stabilised (Figure 2).

These results suggest that there are certain interactions between like-charged particles and ionic surfactants which are crucial for the synergistic effect in emulsion stabilization. One of these may be the electrostatic repulsion between particles and surfactant head groups, as mentioned by Khosravani et al. ${ }^{\text {[3] }}$ who prepared $\mathrm{O} / \mathrm{W}$ emulsions using synthetic alumina particles with various surfactants and found that among an anionic, a cationic and a nonionic surfactant only CTAB addition resulted in finer and more stable emulsions. Electrostatic repulsion promotes surfactant adsorption to the oil-water interface particularly at low surfactant concentration (reducing IFT slightly). ${ }^{[19]}$ Although the monolayer of ionic surfactant at the oilwater interface is far from saturated at such low concentrations, it endows droplets with charge resulting in electrostatic repulsion between droplets and between droplets and particles preventing drop flocculation. We note that no enhancement in emulsion stability was seen in mixtures of nonionic surfactant $\left(\mathrm{C}_{12} \mathrm{E}_{10}\right)$ and alumina particles, pointing to the importance of electrostatic repulsion in this phenomenon. Another factor may be that particles jam in the aqueous lamellae between droplets forming a physical obstacle preventing coalescence of droplets as seen in Figure 4. The aqueous lamellae loaded with particles may 
have an increased viscosity and reduce the kinetics of water drainage. This has been reported previously for aqueous foams [30-32, 34] where particles larger than a critical size can be captured in the Plateau borders and become jammed once the lamella thickness becomes smaller than the particle diameter through drainage. The viscosity in the lamella then increases sharply inhibiting drainage. [34-36] In our systems, however, the bulk particle concentration can be as low as $10^{-4}$ wt.\% and the emulsions resulting after creaming are all fluid-like as shown in Fig. S12. Moreover, the viscosity of an aqueous dispersion containing the highest alumina particle concentration used $(0.5$ wt.\%) is similar to that of pure water. Thus the viscosity increase in the aqueous lamellae may be limited and the particle volume fraction may not increase sufficiently for jamming to occur.

Since the particles are charged similarly to surfactant molecules adsorbed at the interface, we suggest that at low concentration they disperse in the aqueous lamellae between surfactant-coated drops due to electrostatic repulsion. The Debye length varies between $304 \mathrm{~nm}$ at $0.001 \mathrm{mM} \mathrm{CTAB}$ (> dav) and $30 \mathrm{~nm}$ at $0.1 \mathrm{mM}$ CTAB $\left(<\mathrm{d}_{\mathrm{av}}\right.$ excluding particles from thin films) with surfactant regarded as a 1:1 electrolyte. In this way the thickness of the lamellae can be significantly larger than that in the absence of particles enhancing the stability of the emulsion to flocculation and coalescence. This is supported by the SEM images shown in Figure 4 (A-2) where the lamellae between droplets are relatively thick. This can explain why $\mathrm{C}_{\text {min,Al2O3 }}$ increases with increasing $\mathrm{CTAB}$ concentration to the right of Figure 3(a). Since the total oil-water interfacial area increases due to a reduction in average droplet size, the particle and surfactant concentrations should therefore be increased correspondingly to achieve a minimum lamella thickness to prevent coalescence. This idea is also supported by the occurrence of demulsification upon adding $3 \mathrm{mM} \mathrm{Na}_{3} \mathrm{PO}_{4}$ (Fig. S13) which acts to screen electrostatic repulsions, and by the switching property of emulsions shown in Figure 6 where once an equimolar amount of anionic surfactant SDS is added into the emulsion, demulsification occurs due to ion pair formation between both surfactants which destroys the electrical repulsion. ${ }^{[27]}$ If free cationic surfactant is subsequently added, the electrical repulsion is re-established and a stable emulsion is re-formed following homogenization.

In summary, we propose a novel stabilization mechanism of $\mathrm{O} / \mathrm{W}$ emulsions containing very low concentrations of charged surfactant and like-charged nanoparticles. Emulsion droplets are sparsely coated by ionic surfactant molecules with similarly charged nanoparticles dispersed in the continuous phase. The adsorption of surfactant at the oil-water interface, which is slightly enhanced by particles due to electrostatic repulsion, reduces the IFT and endows droplets with charge such that they repel other droplets and particles. Charged particles dispersed in water enable sufficient lamella thickness reducing water drainage and hindering flocculation and coalescence of droplets. In comparison with either Pickering emulsions or conventional emulsions the novel emulsions described here are scientifically fascinating and economically significant for practical applications.

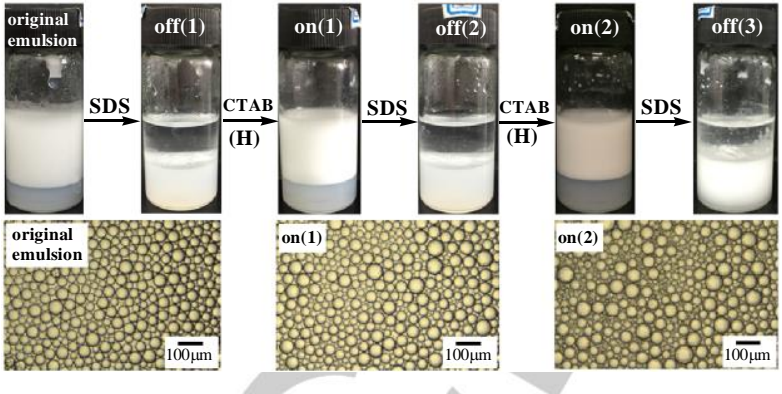

Figure 6. Appearance and micrographs of $n$-decane-in-water emulsions stabilised by 0.5 wt.\% alumina nanoparticles plus $0.3 \mathrm{mM} \mathrm{CTAB}$, followed by switching off via addition of $0.3 \mathrm{mM}$ SDS and switching on via addition of 0.3 $\mathrm{mM}$ CTAB with homogenization $(\mathrm{H})$ successively, taken $24 \mathrm{~h}$ after operation.

\section{Acknowledgements}

Financial support from the National Natural Science Foundation of China (NSFC 21573096, 21473080) and from MOE \& SAFEA for the 111 Project (B13025) is gratefully acknowledged.

Keywords: Alumina nanoparticle $\cdot \mathrm{CTAB} \cdot$ Electrical repulsion • Emulsion stabilization

[1] P. Becher, Encyclopedia of Emulsion Technology, Vol. 1, Marce Dekker, New York and Basel, 1983.

[2] M.J. Rosen, J.T. Kunjappu, Surfactants and Interfacial Phenomena, 4th edition, Wiley, Hoboken, 2012.

[3] R. Aveyard, B.P. Binks, J.H. Clint. Adv. Colloid Interface Sci. 2003, 100 503-546.

[4] B.P. Binks, T.S. Horozov, in Colloidal Particles at Liquid Interfaces (Eds.: B.P. Binks, T.S. Horozov), Cambridge University Press Cambridge, 2006, Chapter 1.

[5] Y. Chevalier, M.A. Bolzinger, Colloids Surf. A 2013, 439, 23-34

[6] B.P. Binks, P.D.I. Fletcher, Langmuir 2001, 17, 4708-4710.

[7] A. Perro, S. Reculusa, S. Ravaine, E. Bourgeat-Lami, E. Duguet, J. Mater. Chem. 2005, 15, 3745-3760.

[8] V.N. Paunov, O. Cayre, Adv. Mater. 2004, 16, 778-791.

[9] J.W. Kim, D. Lee, H.C. Shum, D.A. Weitz, Adv. Mater. 2008, 20, 32393243

[10] A. Walther, M. Hoffmann, A.H. Müller, Angew. Chemie 2008, 120, 723726.

[11] S. Jiang, Q. Chen, M. Tripathy, E. Luijten, K.S. Schweizer, S. Granick, Adv. Mater. 2010, 22, 1060-1071

[12] S. Fujii, Y. Yokoyama, Y. Miyanari, T. Shiono, M. Ito, S. Yusa, Y Nakamura, Langmuir 2013, 29, 5457-5465.

[13] N. Glaser, D.J. Adams, A. Böker, G. Krausch. Langmuir 2006, 22, 5227-5229.

[14] B.P. Binks, Curr. Opin. Colloid Interface Sci. 2002, 7, 21-24.

[15] Z.G. Cui, K.Z. Shi, Y.Z. Cui, B.P. Binks, Colloids Surf. A 2008, 329, 6774.

[16] A. Tsugita, S. Takemoto, K. Mori, T. Yoneya, Y. Otani, J. Colloid Interface Sci. 1983, 95, 551-560.

[17] X. Zhai, S. Efrima, J. Phys. Chem. 1996, 100, 11019-11028.

[18] J.T. Tang, P.J. Quinlan, K.C. Tam, Soft Matter 2015, 11, 3512-3529.

[19] D. Arab, A. Kantzas, S.L. Bryant. J. Petrol. Sci. Eng. 2018,163, 217-242.

[20] Q. Liu, S. Zhang, D. Sun, J. Xu. Colloids Surf. A 2010, 355, 151-157.

[21] Q. Lan, F. Yang, S. Zhang, S. Liu, J. Xu, D. Sun, Colloids Surf. A 2007, 302, 126-135

[22] Z.G. Cui, Y.Z. Cui, C.F. Cui, Z. Chen, B.P. Binks, Langmuir 2010, 26 , 12567-12574. 
[23] B.P. Binks, J.A. Rodrigues, Angew. Chem. Int. Ed. 2007, 46, 53895392.

[24] Z.G. Cui, L.L. Yang, Y.Z. Cui, B.P. Binks, Langmuir 2010, 26, $4717-$ 4724.

[25] J.Z. Jiang, Y. Zhu, Z.G. Cui, B.P. Binks, Angew. Chem. Int. Ed. 2013 , 52, 12373-12376.

[26] M.D. Xu, W.Q. Zhang, X.M. Pei, J.Z. Jiang, Z.G. Cui, B.P. Binks, RSC Adv., 2017, 7, 29742-29751.

[27] Y. Zhu, J.Z. Jiang, K.H. Liu, Z.G. Cui, B.P. Binks, Langmuir 2015, 31, 3301-3307.

[28] K.H. Liu, J.Z. Jiang, Z.G. Cui, B.P. Binks, Langmuir 2017, 33, 22962305.

[29] Y.M. Zhang, S. Guo, X.F. Ren, X.F. Liu, Y. Fang, Langmuir 2017, 33, 12973-12981.

[30] R. Aveyard, B.P. Binks, P.D.I. Fletcher, C.E. Rutherford. J. Disp. Sci. Technol. 1994, 15, 251-271.

[31] H. Ma, M.X. Luo, L.L. Dai, Phys. Chem. Chem. Phys. 2008, 10, $2207-$ 2213.

[32] Q. Sun, Z.M. Li, J.Q. Wang, S.Y. Li, B.F. Li, L. Jiang, H.Y. Wang, Q.C. Lü, C. Zhang, W. Liu, Colloids Surf. A 2015, 471, 54-64.

[33] S. Khosravani, M. Alaei, A.M. Rashidi, A. Ramazani, M. Ershadi, Mater. Res. Bull. 2013, 48, 2186-2190.

[34] B. Haffner, Y. Khidas, O. Pitois. Soft Matter 2014, 10, 3277-3283.

[35] Y. Khidas, B. Haffner, O. Pitois. Soft Matter 2014, 10, 4137-4141.

[36] F. Rouyer, B. Haffner, N. Louvet, Y. Khidas, O. Pitois. Soft Matter 2014, 10, 6990-6998. 
Entry for the Table of Contents (Please choose one layout)

\section{COMMUNICATION}

Novel oil-in-water emulsions are prepared which are stabilised by a cationic surfactant in combination with similarly charged alumina nanoparticles at extremely low concentrations. The stabilization mechanism is revealed which is applicable to different oils and is universal for positively charged particles with cationic surfactant and negatively charged particles with anionic surfactant.

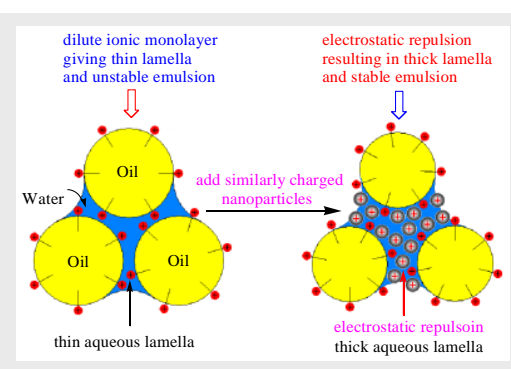

M. D. Xu, J. Z. Jiang, X. M. Pei, B. L. Song, Z. G. Cui ${ }^{\star}$ and B. P. Binks*

Page No. - Page No.

Novel Oil-in-Water Emulsions Stabilised by lonic Surfactant and Similarly Charged Inorganic Nanoparticles at Very Low Concentrations 